\title{
Efficacité comparée de la synchronisation de l'oestrus par la progestérone et la PGF2 $\alpha$ chez les chèvres naines guinéennes du Cameroun
}

\author{
J.R. KANA*, F. NGOULA, A. KENFACK, F. TENDONKENG et T.E. PAMO \\ Département des Productions Animales, Faculté d'Agronomie et des Sciences Agricoles, Université de \\ Dschang, BP 188 Dschang, Cameroun. \\ *Auteur correspondant, E-mail: kanajean@yahoo.fr, Tel: +237 74893394
}

\section{RESUME}

La synchronisation des chaleurs chez la chèvre naine de Guinée a été appréciée en zone périurbaine de Dschang au Cameroun. Les observations ont été menées sur deux lots de 12 chèvres non gestantes pesant en moyenne 12,5 $\pm 2,37 \mathrm{~kg}$ constitués respectivement à la Ferme d'Application et de Recherche de l'Université de Dschang et en milieu paysan. Dans chacun de ces lots, 6 animaux étaient soumis à un traitement à la progestérone et les 6 autres à la prostaglandine F2 $\alpha$. (Lutalyse ${ }^{\mathrm{TM}}$, Dinoprost thromethamine). La progestérone (acétate de médroxyprogestérone) était administrée à travers les éponges intra vaginales qui étaient maintenues pendant 20 jours et la prostaglandine F2 $\alpha$ a été administrée en une seule dose par injection intramusculaire à la dose de $2,5 \mathrm{mg} /$ animal soit $0,5 \mathrm{ml} / 10 \mathrm{~kg}$ de poids vif. Le taux d'induction de l'oestrus observé en milieu paysan était de $50 \%$ chez les animaux traités à la progestérone et à la prostaglandine. Entre 48 et 72 heures après le retrait des éponges et l'injection de la prostaglandine, ces animaux ont présenté un corps jaune actif matérialisé par la sécrétion importante de la progestérone. Dans tous les cas, la concentration de la progestérone était très élevée (>23 ng/ml) au cours de la phase lutéale qui a suivi l'oestrus induit respectivement chez les animaux traités à la prostaglandine et à la progestérone indiquant la bonne qualité des follicules et des corps jaunes actifs. Par contre ces traitements étaient inopérants chez tous les animaux en station. Ils ont présenté un taux de progestérone inférieur à $1 \mathrm{ng} / \mathrm{ml}$. Le taux de progestérone sérique des animaux traités en milieu paysan était significativement $(\mathrm{p}>0,05)$ plus élevé que celui des animaux traités en station. Dans l'ensemble, cette étude a révélé une faible efficacité d'induction de l'oestrus par des traitements hormonaux chez les chèvres naines de Guinée en station.

(C) 2008 International Formulae Group. All rights reserved.

Mots clés : Chèvre naine de Guinée, Progestérone, Prostaglandine F2 $\alpha$, Induction de l'oestrus, Synchronisation des chaleurs, Cameroun.

\section{INTRODUCTION}

$\mathrm{La}$ faible productivité des petits ruminants généralement observée en régions tropicales est imputable à plusieurs facteurs parmi lesquels la nutrition, les maladies et leur faible potentiel génétique (Buvanendra, 1989). L'utilisation de l'insémination artificielle peut être un moyen efficace pour augmenter les performances de reproduction des ruminants adaptés à la zone tropicale soudano guinéenne (Lhoste et al., 1993). Les problèmes associés à la détection des chaleurs constituent un handicap majeur à l'expansion de l'insémination artificielle dans cette région (Zongo et al., 2001). La synchronisation des oestrus pourrait permettre de grouper les mises bas selon les disponibilités alimentaires et à des fins de satisfaction de la demande sur le marché tout en respectant le calendrier agricole de la région qui de plus en plus développe les cultures de contre saison.

L'accroissement du nombre des unités de production relativement importantes, la disponibilité des techniques d'insémination artificielle et l'avantage économique représenté par la possibilité de réaliser les 
mises bas au moment le plus favorable, font que la planification de la reproduction grâce à la maîtrise de la synchronisation de l'oestrus s'avère souhaitable sinon indispensable.

La nécessité d'acquérir les données de base sur l'application de la biotechnologie chez la chèvre naine de Guinée et une meilleure maîtrise de ses performances de reproduction dans la perspective d'une intensification de sa production nous a conduits à initier cette étude dont l'objectif principal est de synchroniser les chaleurs en vue de programmer les mise-bas à une période de l'année ou les conditions climatiques sont favorables et les ressources alimentaires abondantes et de bonne valeur nutritive.

\section{MATERIEL ET METHODES \\ Zone d'étude}

La présente étude a été menée en milieu paysan en zone périurbaine de la ville de Dschang, dans les hautes terres de l'Ouest du Cameroun et en station, à la Ferme d'Application et de Recherche (FAR) de l'Université de Dschang. La localité est située à $05^{\circ} 26^{\prime}$ latitude Nord, $10^{\circ} 26^{\prime}$ longitude Est et à une altitude moyenne de $1420 \mathrm{~m}$. Le climat de la région est équatorial de type Camerounien d'altitude. Les précipitations varient entre 1500 et $2000 \mathrm{~mm}$ par an et les températures oscillent entre $14{ }^{\circ} \mathrm{C}$ (JuilletAoût) et $25^{\circ} \mathrm{C}$ (Février).

L'élevage des petits ruminants dans cette zone est de type semi-intensif avec une durée moyenne de parcours d'environ 9 heures sur pâturage naturel composé essentiellement des graminées (Pennisetum spp, le Brachiaria spp...), des légumineuses herbacées (Desmodium spp, Stylosantes spp...), des ligneux et des divers fourrager. A la FAR, le parcours est essentiellement dominé par les graminées dont les principaux sont le Brachiaria ruziziensis et le Panicum maximum.

\section{Animaux et traitements}

Vingt et quatre chèvres naines de Guinée (Capra reversa) divisées en deux lots de douze animaux chacun ayant fait au moins une mise bas ont été respectivement constitués chez les paysans en zone périurbaine de la ville de Dschang et en station à la FAR de l'Université de Dschang. En station, les animaux étaient libres au parcours entre 8 heures le matin et 17 heures le soir. Au début de l'essai ces animaux étaient vaccinés contre la Peste des petits ruminants (PPR) et ils avaient reçu une couverture d'antibiotique à l'oxitétracycline. En milieu paysan, les animaux n'avaient reçu aucun traitement et ils étaient attachés à la corde sur des piquets dans les parcours toute la journée. Le soir, ils étaient ramenés dans les habitations ou ils passaient la nuit dans des enclos couverts derrière les maisons d'habitations pour les uns et dans un coin dans les habitations pour les autres. Ces animaux ont été divisés en quatre groupes de 6 animaux chacun (deux groupes en station et deux groupes chez les paysans). Ils pesaient individuellement entre 12 et 12,6 $\mathrm{kg}$ avec un poids moyen par groupe de 12,5 \pm $2,37 \mathrm{~kg}$. En station comme en milieu paysan chacun des groupes était soumis au traitement d'induction à l'oestrus par la prostaglandine F2 $\alpha$ (Lutalyse ${ }^{\mathrm{TM}}$, Dinoprost thromethamine) et par les progestagènes (acétate de médroxyprogestérone) respectivement.

Le prélèvement de sang dans la veine jugulaire a été effectué chez tous les animaux tous les 3 jours pendant 9 jours. Par la suite, la prostaglandine a été injectée à la dose de 2,5 mg/animal soit $0,5 \mathrm{ml} / 10 \mathrm{~kg}$ de poids vif. Après cette injection le prélèvement du sang a eu lieu toutes les 8 heures pendant 3 jours puis tous les 3 jours pendant 21 jours. Chez les animaux traités à la progestérone, la pose de l'éponge intra vaginale contenant $60 \mathrm{mg}$ d'acétate de médroxyprogestérone a été effectuée et le prélèvement du sang a continué tous les 3 jours jusqu'au retrait des éponges après 20 jours. Après le retrait des éponges le prélèvement du sang a eu lieu toutes les 8 heures pendant 3 jours puis tous les 3 jours pendant 21 jours. Le sang prélevé était laissé au repos et le sérum recueilli était conservé au congélateur jusqu'au dosage de la progestérone à la fin de l'étude.

Les taux de la progestérone sérique ont été déterminés suivant le test radioimmunologique (RIA) dont le protocole a été conjointement mis au point par la FAO et l'AIEA (1996).

\section{Analyse statistique}

Les résultats ont été exprimés en moyenne \pm écart type et les différences ont été considérées comme significatives au seuil de 
probabilité de 5\%. Les moyennes ont été séparées par le test " $\mathrm{t}$ " de Student.

\section{RESULTATS}

En station tous les animaux sont restés réfractaires au traitement à la progestérone et à la prostaglandine (Table 1). Le dosage radio immunologique de leurs sérums a montré des traces de progestérone dont la moyenne était inférieure à $1 \mathrm{ng} / \mathrm{ml}$ avant et après le traitement traduisant le manque d'un corps jaune de cycle. Par contre en milieu paysan 3 chèvres sur 6 soit $50 \%$ d'animaux ont respectivement répondu positivement au traitement à la progestérone (Figure 1) et à la prostaglandine (Figure 3). Juste 72 heures après le retrait des éponges, on a noté une production massive et croissante de la progestérone. De même 72 heures après l'injection de la prostaglandine, on a noté une production progressive de la progestérone qui a atteint son pic à $23,83 \pm 0,05 \mathrm{ng} / \mathrm{ml}$ en moyenne au bout de 15 jours (Figure 2). Après ce pic une baisse progressive de la courbe de production de la progestérone a été notée jusqu'à la fin de notre étude au $34^{\mathrm{e}}$ jour du prélèvement. Dans l'ensemble le taux de progestérone sérique des animaux traités en milieu paysan était significativement $(p>0,05)$ plus élevé que celui des animaux traités en station.

Pendant le séjour des éponges dans le vagin des animaux en station comme en milieu paysan le taux de la progestérone est resté en moyenne inférieur à $1 \mathrm{ng} / \mathrm{ml}$. Chez les animaux ayant répondu au traitement après le retrait des éponges, le taux de la progestérone sérique était en moyenne de 24,93 $\pm 2,08 \mathrm{ng} / \mathrm{ml}$ à la fin de notre essai.

\section{DISCUSSION}

$\mathrm{Au}$ cours de cette étude, le taux d'induction de l'oestrus chez les chèvres par la progestérone ou par la Prostaglandine en station était nul. Cette observation est contraire aux observations faites par Cognie et al. (1984) et Baril et al. (1993). Cela pourrait être en liaison avec le petit format des femelles de cette race soumises à un traitement préconisé pour les races de grand format européennes.

La forte imprégnation hormonale qui résulte du traitement, notamment la progestérone, est bien reconnue (Aguer, 1981; Lhoste et al., 1993; Baril et al., 1993; Zongo et al., 2001) pour raccourcir le délais d'induction de l'oestrus, et expliquerait les chaleur survenant 72 heures après le retrait des éponges chez la chèvre naine de Guinée.

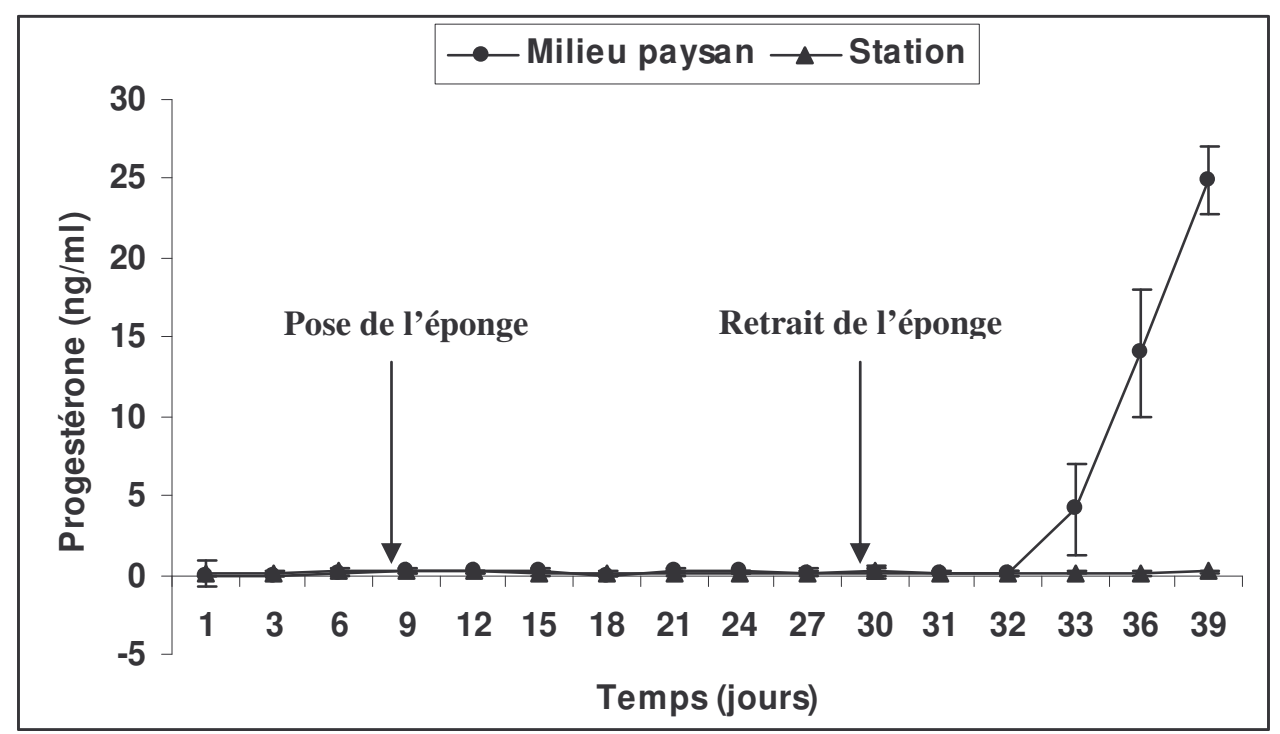

Figure 1: Cinétique de la progestérone chez les CNG traitées à la progestérone en milieu paysan et en station. 


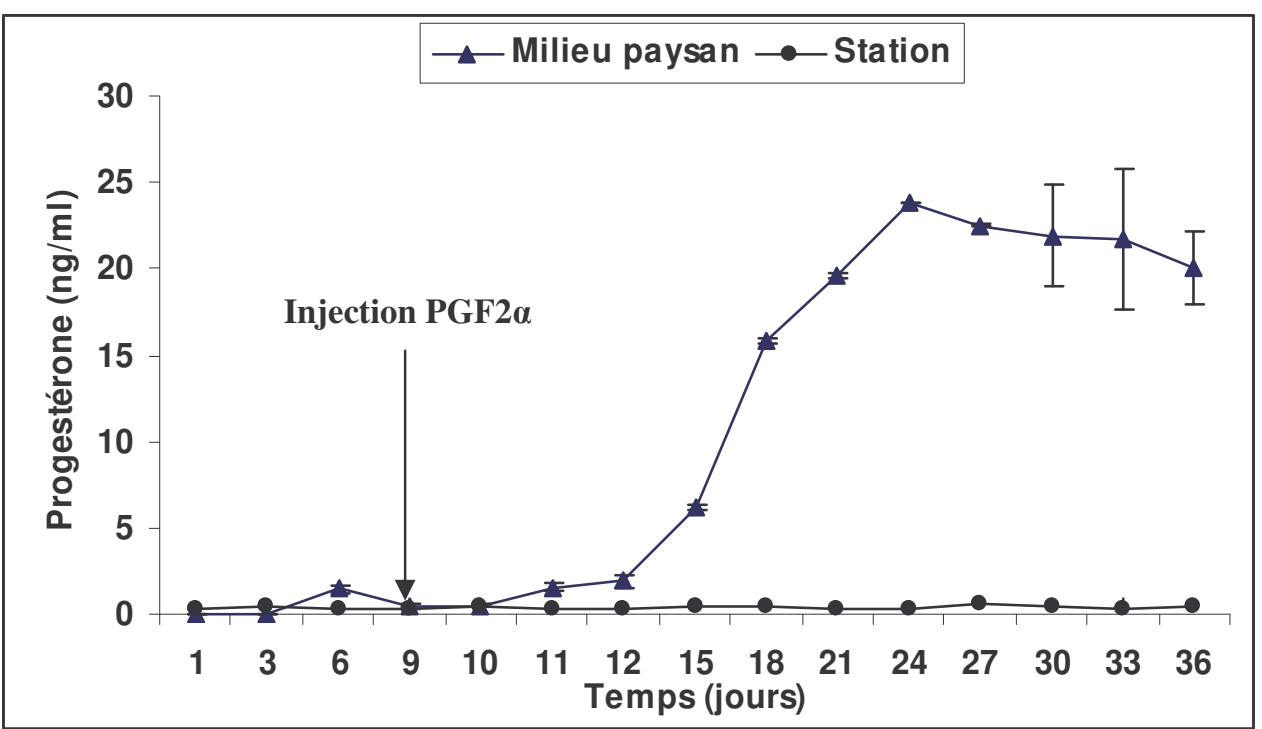

Figure 2: Cinétique de la progestérone chez les CNG traitées à la PGF2 $\alpha$ en milieu paysan et en station.

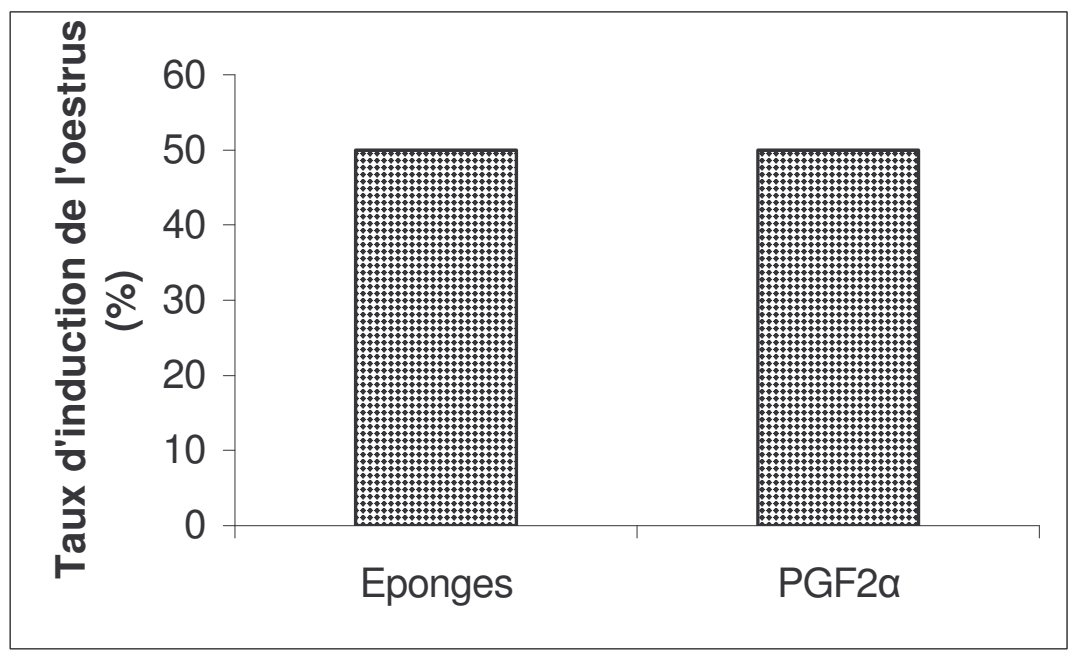

Figure 3: Taux d'induction de l'œstrus chez la CNG 96 heures après l'injection de la PGF2 $\alpha$ et le retrait des éponges en milieu paysan.

Il en est de même pour la prostaglandine dont les effets de resserrement des délais d'apparition des chaleurs ont été montrés par Steffan (1981), Macmillan (1983) et Fetrow et Blanchard (1987).

Les effets de resserrements des délais d'apparition des chaleurs par la prostaglandine et par la progestérone tels que nous avons noté dans cette étude ont été également montrés par Thimonier et al. (1984), Eddy et Med, (1999). Dans les conditions de routine, les prostaglandines et les éponges imprégnées de progestérone sont utilisées avec succès en association (Baril et al., 1993). Le fait de les avoir utilisé individuellement pourrait expliquer le faible taux d'induction de 
Tableau 1 : Evolution du taux de la progestérone sérique (ng/ml) chez la CNG après le traitement aux progestagènes et à la PGF2 $\alpha$.

\begin{tabular}{|c|c|c|c|c|c|c|c|c|}
\hline \multirow{2}{*}{ Traitements } & \multicolumn{8}{|c|}{ Temps (jours) } \\
\hline & & $\mathbf{0}$ & 3 & 6 & 9 & 12 & 15 & 18 \\
\hline \multirow{2}{*}{ Eponges } & Milieu Paysan & $0,12 \pm 0,34 a$ & $4,12 \pm 2,8 \mathrm{a}$ & $14 \pm 4,05 a$ & $24,93 \pm 2,08 \mathrm{a}$ & $25,86 \pm 3,04 \mathrm{a}$ & $26 \pm 4,20 \mathrm{a}$ & $25,93 \pm 3,02 a$ \\
\hline & Station & $0,22 \pm 0,29 a$ & $0,13 \pm 0,07 b$ & $0,10 \pm 0,11 b$ & $0,22 \pm 0,11 b$ & $0,32 \pm 0,12 b$ & $0,23 \pm 0,04 b$ & $0,30 \pm 0,12 b$ \\
\hline \multirow{2}{*}{ PGF2 $\alpha$} & Milieu Paysan & $0,43 \pm 0,17 \mathrm{a}$ & $1,92 \pm 0,34 \mathrm{a}$ & $6,19 \pm 0,17 a$ & $15,86 \pm 0,13 \mathrm{a}$ & $19,6 \pm 0,12 \mathrm{a}$ & $23,83 \pm 0,05 a$ & $22,53 \pm 0,63 a$ \\
\hline & Station & $0,29 \pm 0,29 a$ & $0,34 \pm 0,15 b$ & $0,38 \pm 0,17 b$ & $0,47 \pm 0,15 b$ & $0,30 \pm 0,17 b$ & $0,36 \pm 0,46 b$ & $0,63 \pm 0,18 b$ \\
\hline
\end{tabular}

a,b: Les moyennes de la même colonne et pour le même traitement suivies par la même lettre ne diffèrent pas entre elles au seuil de $5 \%$. 
l'oestrus noté dans cette étude. Dans ces conditions, chez les chèvres en anoestrus les taux d'hormones utilisés lors des traitements ne seront pas suffisants pour induire l'ovulation (Cognie et al., 1984). Selon Thimonier et al. (1984), l'injection de la prostaglandine juste après le retrait de l'éponge entraînerait la lyse des corps jaunes persistants et favoriserait la précision de la synchronisation des chaleurs dans le troupeau. En effet, les prostaglandines sont efficaces pour synchroniser les oestrus seulement s'il y a présence d'un corps jaune du cycle (Baril et al., 1993).

Dans notre étude, le taux de la progestérone noté après le traitement à la prostaglandine ou à la progestérone était très élevé par rapport aux taux naturels rapportés par Baril et al. (1993) pendant la période postovulatoire et pendant la gestation chez les chèvres. De même ce taux était largement supérieur à celui rapporté par Zongo et al. (2001) chez les Zébus Azawak et les taurins Gourunsi après induction de l'oestrus aux progestagènes combinés à la prostaglandine et à la PMSG. Dans les conditions environnementales tropicales de nombreux auteurs ont signalé des cycles sexuels irréguliers (Lhoste et al., 1993) et une fréquence élevée de suboestrus (Thimonier et al., 1984) chez les petits ruminants. Ceci pourrait expliquer le fort taux de traitements inopérants dans notre étude. Aussi, les perturbations des femelles au moment de l'apparition des chaleurs après les traitements hormonaux telles que les perturbations de l'environnement et les prises fréquentes de sang peuvent retarder l'ovulation de plus de 24 heures (Cognie et al., 1984).

\section{Conclusion}

Dans son ensemble, cette étude a révélé une faible efficacité d'induction de l'oestrus par des traitements hormonaux chez les chèvres naines de Guinée en station et en milieu paysan. Cette difficulté à regrouper les chaleurs pourrait être un handicap majeur à l'application des techniques de la biotechnologie telle que l'insémination artificielle chez les chèvres de petites tailles à faible potentiel génétique. Cependant, la concentration très élevée de la progestérone (>23 ng/ml) au cours de la phase lutéale qui a suivi l'oestrus induit respectivement chez les animaux traités à la prostaglandine et à la progestérone pourrait être une présomption de la bonne qualité des follicules et des corps jaunes actifs.

\section{REMERCIEMENTS}

Les auteurs adressent leurs remerciements à l'AIEA, à l'Université de Dschang et à toute l'équipe du Laboratoire de Physiologie Animale de la Faculté d'Agronomie et des Sciences Agricoles de l'Université de Dschang pour leur contribution à la réalisation de ce travail.

\section{REFERENCES BIBLIOGRAPHIQUES}

Adebambo OA, Samuel AO, Anakade AD. 1994. Causes of variation in reproductive performance of West Africa Dwarf goat. Nig J. of Ani. Prod., 1-2: 29-36.

Aguer D. 1981. Les progestagènes dans la maîtrise des cycles sexuels chez les bovins. Recl. Med. Vét., 157: 53-60.

Baril G, Chemineau P, Cognie Y, Leboeuf B, Orgeur P, Vallet JC. 1993. Manuel de formation pour l'insémination artificielle chez les Ovins et les Caprins. FAO; 187p.

Buvanendra V. 1989. Constraints to improving reproductive efficiency of ruminant livestock in Developping Countries. Proceed, Feeding strategies for improving productivity of ruminant livestock in Developping Countries. IAEA Vienna; 51-61.

Cognie Y, Schirar A, Martinet J, Poulin N, Mirman B. 1984. Activité reproductive et maîtrise de l'ovulation chez les brebis $\left(9^{\mathrm{e}}\right.$ édn). Journées de Recherche Ovine et Caprine; 109-133.

Eddy RG, Med FRCVS. 1999. Hormonal control of reproduction. Normal nonpregnant female. Lecture note, Diploma course in bovine reproduction, The University of Liverpool.

FAO/IAEA. 1996. Progesterone RIA Kit. Joint FAO/IAEA Programme in Animal Production and Health.

Fetrow J, Blanchard T. 1987. Economy impact of the use of prostaglandin to induce estrous in dairy cows. J. Am. Vet. Med. Ass., 190: 163-169.

Lhoste P, Dolle V, Rousseau J, Soltner D. 1993. Manuel de zootechnique des régions chaudes. Les systèmes d'élevage. Collection Précis d'Elevage; 220p. 
Macmillan KL. 1983. Prostaglandin responses in dairy herd breeding programmes. New Zel. Vet. J., 31: $110-113$.

Steffan J. 1981. Applications thérapeutiques et zootechniques de la prostaglandine F2 $\alpha$ chez les bovins. Recl. Méd. Vét., 157: 6169.

Thimonier J, Chemineau P, Gauthier D. 1984. Augmenter la fertilité des ruminants en zone tropicale: une réalité. Reproduction des ruminants en zone tropicale, Point à
Pitre (FWI) 8-10 Juin 1983. Les Colloques de l'INRA. No 20, pp.400-413. Zongo M, Pitala W, Boly H, Sulon J, Noelita MS, Leroy PL, Beckers JF, Sawadogo L. 2001. Profil de la progestérone des vaches zébu 'Azawak' et taurins 'Gourunsi' après induction de l'oestrus aux progestagènes combinés à la prostaglandine et à la PMSG. Tropicultura, 19(3): 131-134. 